Research Article

\title{
Thematic Maps of the Impact of Urbanization and Socioeconomic Factors on the Distribution of the Incidence of Cutaneous Leishmaniasis Cases in Sefrou Province, Central North of Morocco (2007-2011)
}

\author{
Fatima Zahra Talbi $\left(\mathbb{D},{ }^{1}\right.$ Nordine Nouayti, ${ }^{2}$ Hajar El Omari ${ }^{\mathbb{D}},{ }^{3}$ Mohamed Najy, \\ Khadija Lahouiti, ${ }^{5}$ Mouhcine Fadil $\left(\mathbb{D},{ }^{6}\right.$ Hassan Ech-Chafay, ${ }^{4}$ Mohamed Lachhab, ${ }^{4}$ \\ Abdellatif Janati Idrissi, ${ }^{1}$ and Abdelhakim El Ouali Lalami ${ }^{1,7}$ \\ ${ }^{1}$ Laboratory of Biotechnology and Preservation of Natural Resources, Faculty of Sciences Dhar El Mahraz, \\ Sidi Mohamed Ben Abdellah University, Fez 30000, Morocco \\ ${ }^{2}$ Applied Sciences Laboratory, Water and Environmental Engineering Team, National School of Applied Sciences, \\ Abdelmalek Essaadi University, Tetouan, Morocco \\ ${ }^{3}$ Natural Resources Management and Development Team, Laboratory of Health and Environment, Faculty of Sciences, \\ Moulay Ismail University, Meknes, Morocco \\ ${ }^{4}$ Laboratory of Agrophysiology, Biotechnology, Environment and Quality, Department of Biology, University Ibn Tofail, \\ Faculty of Science, BP 133, Kenitra 14000, Morocco \\ ${ }^{5}$ Laboratory of Microbial Biotechnology, Department of Biology, Faculty of Sciences and Technology, \\ University Sidi Mohamed Ben Abdellah, BP 2202, Road of Immouzer, Fez, Morocco \\ ${ }^{6}$ Physico-chemical Laboratory of Inorganic and Organic Materials, Materials Science Center (MSC), Ecole Normale Supérieure, \\ Mohammed V University in Rabat, Rabat, Morocco \\ ${ }^{7}$ Higher Institute of Nursing Professions and Health Techniques of Fez, Regional Health Directorate Fez-Meknes, \\ EL Ghassani Hospital, Fez 30000, Morocco
}

Correspondence should be addressed to Fatima Zahra Talbi; fati415189@gmail.com and Abdelhakim El Ouali Lalami; eloualilalami@yahoo.fr

Received 12 January 2020; Revised 8 May 2020; Accepted 19 June 2020; Published 14 July 2020

Academic Editor: Subhada Prasad Pani

Copyright (c) 2020 Fatima Zahra Talbi et al. This is an open access article distributed under the Creative Commons Attribution License, which permits unrestricted use, distribution, and reproduction in any medium, provided the original work is properly cited.

Background. Leishmaniases are vector-borne diseases with health risks. They cause a big health problem. These parasitic diseases are transmitted by the parasite of the genus Leishmania through sandflies. Objective. The aim of this work is to study the distribution of the incidence of cutaneous leishmaniasis (CL) cases and the impact of urbanization and socioeconomic factors and their effects as leishmaniasis risk factors. Methods. We conducted a retrospective study of CL cases collected at the level of Sefrou Province during the period from 2007 to 2011. The data was collected from registers of the Medical Delegation of Sefrou Province. The socioeconomic data, namely, the poverty rate, the popular density, and the type of environment (urban/rural) of Sefrou Province, were obtained from the High Commission for Planning. Statistical analysis was performed by SPSS software (version 20). The data were registered in a Microsoft Excel 2010 file. Statistical analysis was based on one-way analysis of variance (ANOVA), and then a correlation study was carried out (Pearson correlation). The results were considered significant when $p$ was less than 0.05 . The database was analyzed by QGIS 2.18, which is open source software. Results. A total of 349 cases of CL were collected at Sefrou Province from 2007 to 2011. A percentage of $49 \%$ of the cases come from urban areas, while $51 \%$ of the cases come from rural areas. In the statistical analysis, the division of the incidence of CL cases was found to be significantly associated only with urbanization. For the other factors, the number of people or the poverty rate is not taken into account in the incidence dynamics. Conclusion. This study may be useful for the implementation of future adequate measures and controls. Getting rid of leishmaniasis requires a comprehensive approach by acting on the sources of contamination through good continuous surveillance, appropriate management, effective vector control, and awareness-raising strategies. 


\section{Introduction}

Leishmaniasis is a vector-borne disease caused by a flagellated protozoan of the genus Leishmania belonging to the family of Trypanosomatidae, which affects a variety of human and animal populations worldwide [1]. The epidemiology of leishmaniasis is very complex: 20 species of Leishmania are pathogenic to humans, and 30 species of sandflies are proven vectors in the world [2]. It is widespread in all continents with the exception of Oceania. Eighty-eight countries are affected in the Old World and twenty-two countries in the New World [3]. The global prevalence is estimated at 12 million human cases, with an annual incidence of 1.5 to 2 million new cases, and 350 million people would be exposed to the risk of Leishmania transmission [4]. This alarming situation is of paramount importance since Leishmania/HIV coinfections are frequent, and immunocompromised patients are much less controlled, which increases mortality [5]. In Morocco, leishmaniasis is endemic; the Ministry of Health has declared an annual average of 4,076.6 cases of cutaneous leishmaniasis (CL) and a hundred cases of visceral leishmaniasis (VL) in recent years [6]. In the majority of countries around the Mediterranean, leishmaniasis diseases represent entities of great clinical and epidemiological diversity and constitute a real health problem [7]. They can be illustrated in three epidemiological entities: zoonotic cutaneous leishmaniasis (ZCL) with Leishmania major (L. major), anthroponotic cutaneous leishmaniasis (ACL) with Leishmania tropica (L. tropica), and VL with Leishmania infantum (L. infantum) [8]. The geographical position of Morocco is characterized by different ecological and bioclimatic conditions which can influence the diversity of vectors and consequently the distribution of the disease [9]. The choice of Province Sefrou as a study area is justified by its epidemic situation of leishmaniasis, as it is considered among the main foci, by means of its proximity to other important foci such as Taza [10], Sidi Kacem [11], and Errachidia [12]. Several entomological studies were pursued at the Province Sefrou that shows the presence of sandflies with a significant density [13-15]. The spread of cutaneous leishmaniasis disease is linked to various risk factors [16]; among these factors we notice the socioeconomic factors and the urbanization ones $[17,18]$. For several years, risk mapping has been used for several researches based on remote sensing and Geographic Information Systems (GIS). Indeed, these tools are of supreme interest with regard to epidemiological surveillance and the relationship between the spread of the disease and environmental factors [19-21]. For an effective control, it is necessary to establish a risk map of CL from different municipalities in Sefrou Province. This work is a combination of the retrospective study and the risk factors for the distribution of CL: climate, urbanization, poverty, and population. The use of GIS allows us to draw up thematic maps identifying areas at risk. Cartographic analysis is very important to better elucidate the relationship between the distribution of CL disease and the factors of leishmaniasis risk. The aim of this present work is to study the epidemiological situation of this type of parasitosis, the distribution of the incidence, and the impact of urbanization and socioeconomic factors as leishmaniasis risk factors.

\section{Materials and Methods}

2.1. StudyZone. Sefrou Province (Central North of Morocco) covers an area of $3,520 \mathrm{~km}^{2}$ with a predominantly rural population of 259,254 inhabitants. From a geographical point of view, it is limited to the south by the Boulemane and Ifrane Provinces, to the east by the Taza Province, to the north by the Fez prefecture, to the west by the Moulay Yacoub and El Hajeb Provinces, and to the northwest by the Taounate Province [22]. According to the municipal division, Sefrou Province has 5 municipalities and 18 rural communes. Most of these mountainous provinces are contaminated and considered as places of human CL [23].

2.2. Data Sources. The data were collected from the registers of the health directorate of Sefrou Province. All cases of CL recorded on the survey forms were confirmed by parasitological diagnosis direct. CL patients with clinical lesions of CL were passively received during the study period at the local laboratory of the health center of Sefrou Province. [24]. The period of the study was four years, from 2007 to 2011. During this period, a total of 349 cases of CL were the subjects of a retrospective study. The socioeconomic data, namely, the poverty rate, the popular density, and the type of environment (urban/rural) of Sefrou Province, were obtained from the High Commission for Planning [25]. The incidence (I) of CL of each commune was calculated as follows:

$$
I=\frac{\text { number of new cases of the disease in a given population }}{\text { total number of the population at risk of each commune }} \text {. }
$$

2.3. Data Analysis. The data were registered in a Microsoft Excel 2010 file. Statistical analysis was based on one-way analysis of variance (ANOVA), and then the correlation study was carried out (Pearson correlation). The results were considered significant when $p$ is less than 0.05 . To achieve the objective of this study, it is essential to integrate the CL health data into a Geographic Information System in order to analyze its spatial distribution in the various municipalities of the Province of Sefrou. In this respect, the data processing was done by QGIS 2.18 software by the integration of Geographic Information System. Statistical analysis was performed with SPSS software (version 20).

\section{Results and Discussion}

3.1. Epidemiology of Leishmaniasis in Sefrou Province. The temporary evolution of the incidence of CL revealed the endemic nature of this parasitosis in Sefrou Province. The study of the epidemiological situation in this Province during the period from 2007 to 2011 declared 349 cases of CL with an average of 


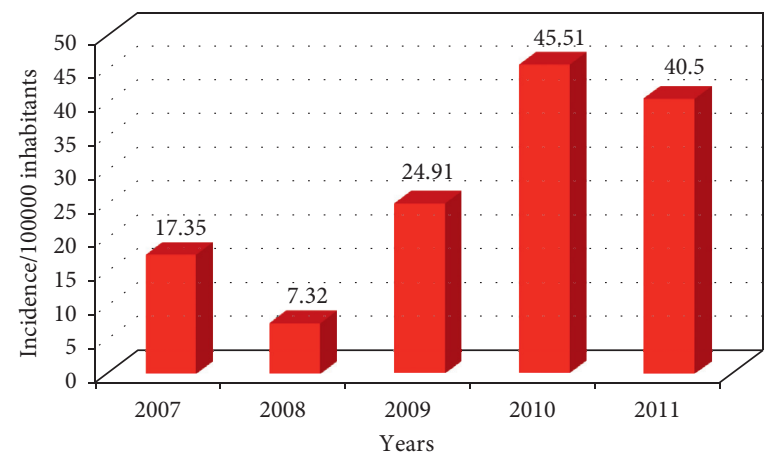

FIgURE 1: Temporal evolution of the annual incidence of CL cases/100,000 inhabitants in Sefrou Province (2007-2011).

TABLE 1: Distribution of the incidence of CL cases according to the urbanization factor.

\begin{tabular}{lcc}
\hline Commune & Incidence 10,000 inhabitant & Type of urbanization \\
\hline Adrej & 31.3 & Rural \\
Aghbalou Aqorar & 13.26 & Rural \\
Ahl Sidi Lahcen & 66.16 & Rural \\
Ain Cheggag & 15.39 & Rural \\
Ain Cheggag & 0 & Rural \\
Ain Timguenai & 0 & Rural \\
Ait Sebaa Lajrouf & 0 & Rural \\
Azzaba & 76.21 & Rural \\
Bir Tam Tam & 18.52 & Rural \\
Dar El Hamra & 0 & Rural \\
Ighzrane & 0 & Rural \\
Kandar Sidi Khiar & 0 & Rural \\
Laanoussar & 2.14 & Rural \\
Mtarnagha & 1.89 & Rural \\
Oulad Mkoudou & 16.62 & Rural \\
Ras Tabouda & 1.53 & Rural \\
Sidi Youssef Ben Ahmed & 0 & Rural \\
Tafajight & 0 & Rural \\
Tazouta & 74.84 & Rural \\
\hline Bhalil & 6.87 & Urbane \\
El Menzel & 66.28 & Urbane \\
Imouzzer Kandar & 0.72 & Urbane \\
Ribate El Kheir & 0 & Urbane \\
Sefrou & 13.62 & Urbane \\
\hline
\end{tabular}

5.77 per 100,000 inhabitants. In 2007, the incidence of CL cases was 17.35 per 100,000 population. This value decreased in 2008 to 10.03 . From 2009, the incidence of cases increased with a peak in 2010 and decreased afterward (Figure 1).

This could be explained by the fact that the situation of this disease has become perturbing, which is linked to the appearance of new foci of transmission where the risk factors persist $[16,26,27]$. Also, the alarming epidemiological situation in 2010 witnessed better management of this public health problem, good awareness, and therefore greater treatment of patients. A cartographic study, on the same province, was able to show that during this year the rural areas were affected by a high rate of incidence (rural municipality of Tazouta and Ahl Sidi Lahcen) [16]. This result corroborates that of the Meknes Province [28] where the incidence varies in time and space; during the same period from 2009 to 2013, the incidence experienced a very marked development, which is justified by unhygienic environmental conditions which increase the risk of leishmaniasis by promoting the multiplication of vectors.

Statistical analysis of this result shows that there is no relationship between the distribution of cases and time (the $p$ value is greater than 0.05 ).

3.2. The Impact of the Urbanization Factor on CL Disease in Sefrou Province. According to the administrative division, Sefrou Province is made up of 24 communes, divided into 7 urban communes and 17 rural communes (Table 1) [25].

According to the census of CL cases, the disease occurs with high incidence in municipalities more than others. We found $49 \%$ of recorded cases of urban origin (Figure 2) with a maximum incidence at El Menzel commune of 66.28 per 10,000 inhabitants. For rural municipalities, $51 \%$ of cases (177 cases) were reported with a maximum 


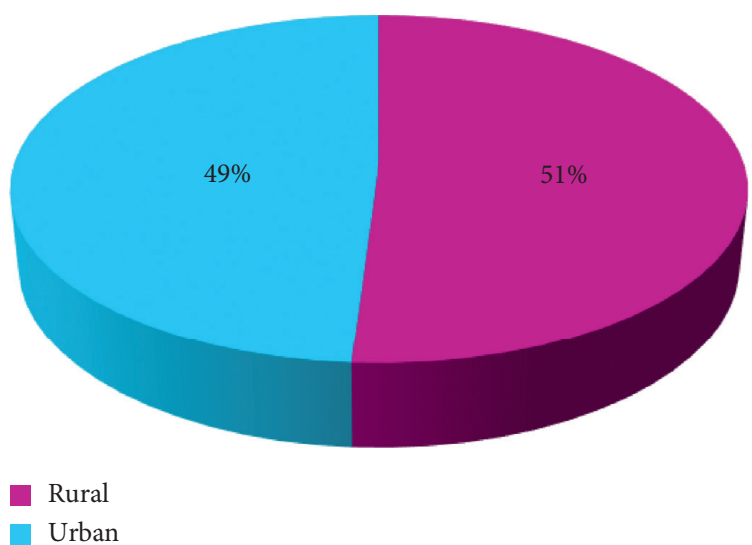

Figure 2: Distribution of the incidence of CL cases according to the state of urbanization of the municipalities of Sefrou Province (2007-2011).

TABLE 2: Test of analysis of variance.

\begin{tabular}{|c|c|c|c|c|c|c|c|}
\hline & & & Sum of squares & $\mathrm{df}$ & Medium square & $F$ & Significance \\
\hline \multirow{3}{*}{ Number of CL cases *urbanization } & Intergroup & Combined & $2,361.604$ & 1 & $2,361.604$ & 4.739 & 0.041 \\
\hline & Intraclass & & $10,465.700$ & 21 & 498.367 & & \\
\hline & Total & & $12,827.304$ & 22 & & & \\
\hline \multirow{3}{*}{ Percentage of CL cases *urbanization } & Intergroup & Combined & 9.415 & 1 & 9.415 & 0.192 & 0.666 \\
\hline & Intraclass & & $1,030.145$ & 21 & 49.055 & & \\
\hline & Total & & $1,039.560$ & 22 & & & \\
\hline
\end{tabular}

${ }^{*}$ Correlation is significant at level 0.05 , df: the degrees of freedom in the source; F: the F-statistic.

incidence at the level of Azzaba commune with 76.21 per 10,000 inhabitants, followed by Tazouta commune $(74.84$ per 10,000 inhabitants) and that of Ahl Sidi Lahcen (66.16 per 10,000 inhabitants).

The analysis of variance revealed a statistically significant difference between the medium numbers of cases from one level of urbanization to another with a confidence rate of 95.0\% $(\mathrm{df}=1, F=4.73, p=0.041)$ Table 2. Accordingly, there is a significant effect between these two variables with a decision of $4.73 \%$.

According to the literature, urbanization interests all continents and all countries [29]. This factor is closely linked to the massive migration to urban areas specifically in the outskirts of cities where there is a lack of waste management, which increases the reproduction of sandflies. In addition, urban CL of the Old World characterizes the anthroponotic type where transmission is carried out through a female sandfly infected with epidermis from one subject to another. This epidemic character is strictly linked to the density of sandflies and the human population. In addition, according to the World Health Organization [30], the phenomenon of urbanization increases the risk of transmission of leishmaniasis especially within an unimmunized population in rural areas. The lack of planning of cities in rural exodus and poor environmental hygiene conditions [31] increase the leishmaniasis risk and lead to favorable bioecological environments to the development of vectors [32-34]. Several studies reveal that urbanization
TABLe 3: Distribution of inhabitants at the level of different municipalities in Sefrou Province (2007-2011).

\begin{tabular}{lc}
\hline Commune & Population \\
\hline Adrej & 2,236 \\
Aghbalou Aqorar & 15,835 \\
Ahl Sidi Lahcen & 5,290 \\
Ain Cheggag & 11,039 \\
Ain Cheggag & 4,286 \\
Ain Timguenai & 2,208 \\
Ait Sebaa Lajrouf & 17,400 \\
Azzaba & 2,493 \\
Bhalil & 11,638 \\
Bir Tam Tam & 9,714 \\
Dar El Hamra & 4,022 \\
El Menzel & 11,465 \\
Ighzrane & 11,050 \\
Imouzzer Kandar & 13,725 \\
Kandar Sidi Khiar & 8,709 \\
Laanoussar & 9,343 \\
Mtarnagha & 5,284 \\
Oulad Mkoudou & 7,821 \\
Ras Tabouda & 6,516 \\
Ribate El Kheir & 12,654 \\
Sefrou & 63,872 \\
Sidi Youssef Ben Ahmed & 11,292 \\
Tafajight & 2,047 \\
Tazouta & 5,745 \\
Zaouia Bougrine & 3,570 \\
Total & 259,254 \\
\hline
\end{tabular}




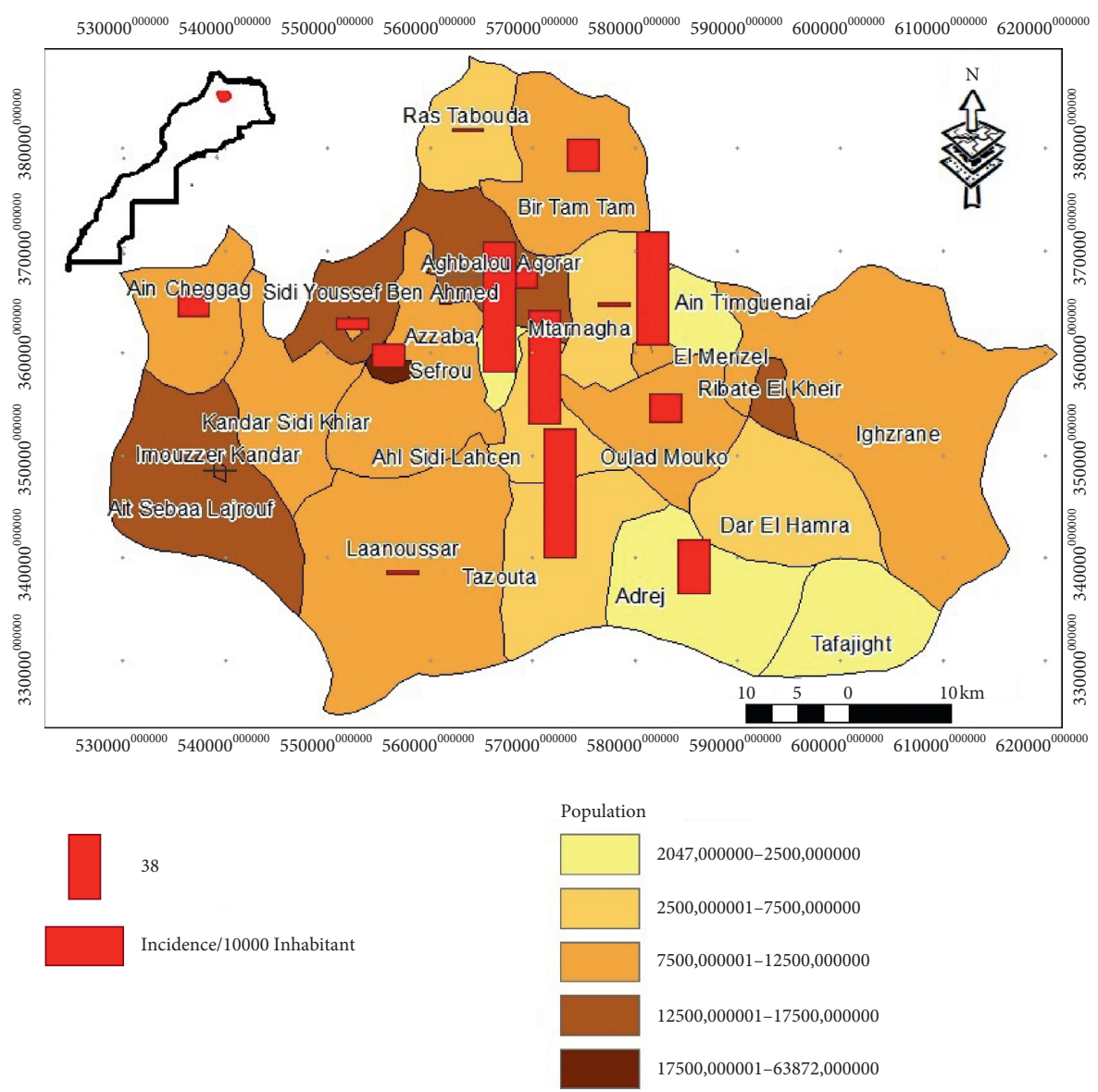

FIGURE 3: Distribution map of the incidence of CL cases of inhabitants at the level of different municipalities in Sefrou Province (2007-2011).

contributes to the increase in the incidence of ACL type $[35,36]$. They also confirmed that the rural areas, where most leishmaniasis patients live, coexist in interresidential homes, or occupy dwellings, allow contact between humans and sandflies and subsequently ensure the circulation of the parasite [27, 37].

\subsection{The Impact of Socioeconomic Factors in Different Municipalities in Sefrou Province on the Distribution of the Incidence of CL Cases}

3.3.1. Distribution of the Incidence of CL Cases according to the Population Factor of Sefrou Province. According to the population census of Sefrou Province [25], the number of inhabitants is 259,254 (Table 3 ).

The distribution of the incidence of CL according to the number of inhabitants of each municipality of Sefrou Province (Figure 3) shows that the municipalities with high incidence rate (Azzaba, Tazouta, El Menzel, and Ahl Sidi Lahcen) have a moderately small number of population.

This distribution of the incidence is not the same at the level of the other communes with low number of population where the incidence rate is low such as commune Ras Tabouda, Bir Tam Tam, and Bhalil. On the other hand, conversely, the commune of Sefrou with its overcrowded situation has been reported to have moderately low incidence (only 13.62 per 10,000 inhabitants) (Figure 3).

According to our result, the majority of municipalities with a high incidence rate are not overcrowded and of rural origin. In addition to the small number of population, the environmental characteristics of these environments make the situation more alarming, which leads to rapid dispersion of cases. Statistical analysis revealed that the distribution of the incidence of CL in this province is not linked to the number of inhabitants by municipality, but to the percentage of cases (Table 4). A positive correlation of 0.474 was confirmed by the normality tests of Kolmogorov-Smirnov ( $p=0.003<$ to 0.05 ) and Shapiro-Wilk $(p=0.001<$ to 0.05$)$, which reveals that the studied samples follow a normal distribution.

From 2007 to 2011, we observed that the number of cases increased with a peak in 2010. This peak could explain the success of the initiative and the management of the disease by the ministry and various authorities. The objectives of this program aim primarily to raise awareness among the population as well as intensifying mass screening activities in schools and localities at risk, following the recommendations of the response plan. Therefore, the mentioned evolution of cases could be explained by the implementation of the strategy for the fight against this disease and the information campaigns conducted by the Ministry of Health according to the national plan for the fight against leishmaniasis, launched in 2009 
TABLE 4: Pearson correlation and significance between incidence of CL cases and the population factor.

\begin{tabular}{lccc}
\hline & & Population & Percentage of CL cases \\
\hline & Pearson correlation & -0.135 & $0.474^{*}$ \\
Incidence per 10,000 population & Sig. (bilateral) & 0.540 & 0.022 \\
& $N$ & 23 & 23 \\
\hline
\end{tabular}

${ }^{*}$ Correlation is significant at level 0.05 (bilateral).



Figure 4: Distribution map of the incidence of CL cases according to poverty factor at the level of different municipalities Sefrou Province (2007-2011).

TABLE 5: Pearson correlation and significance between incidence of CL cases and poverty rate.

\begin{tabular}{|c|c|c|c|}
\hline & & Poverty rate $\%$ & Percentage of CL cases \\
\hline \multirow{3}{*}{ Incidence per 10,000 population } & Pearson correlation & 0.131 & $0.474^{*}$ \\
\hline & Sig. (bilateral) & 0.552 & 0.022 \\
\hline & $N$ & 23 & 23 \\
\hline
\end{tabular}

${ }^{*}$ Correlation is significant at level 0.05 (bilateral).

(Program of Integrated Management of Vector Control). In addition, the factor of the increasing density of the population and the propagation of favorable milieus to the multiplication of the vector could also have an effect on this situation [38]. Indeed, Old World anthroponotic cutaneous leishmaniasis (ACL) requires a reservoir which is humans [15]. This means that the parasite is transmitted from one subject to another through the bite of a peridomestic sandfly, once the infected subjects arrive to the endemic area. Thereby, this can contribute to the extension of its geographical area. We can also explain that the municipalities which have several geographic (proximity to Fez) and economic advantages are highly attractive municipalities. In case of a contamination, overcrowd is directly associated with the high number of CL cases.

3.3.2. Distribution of the Incidence of CL Cases according to the Poverty Factor of Sefrou Province. Figure 4 shows the distribution of the incidence of CL cases according to the poverty rate of different municipalities in Sefrou Province. The 
poverty rate varies from $5.9 \%$ to $26.4 \%$ according to data from the High Commission of Planning.

According to the descriptive analysis, it appears that the incidence of CL does not depend on the poverty rate. Indeed, at the level of the communes of Azzaba (76.21 per 10,000 inhabitants) and Tazouta (74.84 per 10,000 inhabitants), where the incidence is very high compared to the other communes, the poverty rate is ordered, respectively, as $20 \%$ and $17.5 \%$, whereas at the level of the commune of $\mathrm{El}$ Menzel, poverty was only $9.6 \%$ despite the high incidence rate of 66.28 per 10,000 inhabitants (Figure 4).

The statistical analysis of this result shows that there is no link between the distribution of the incidence of CL cases and the poverty rate at the level of Sefrou Province; this is justified by the $p$ value with the order 0.55 which is greater than the significance level of $5 \%$ with a relatively very low correlation coefficient ( $p>0.05, r=0.13$ ) (Table 5).

According to the literature, socioeconomic factors such as poverty and lack of infrastructure seem to be among the main factors that lead to leishmaniasis $[39,40]$. In endemic areas, the leishmaniasis risk is strongly linked to the bad hygienic conditions of the environment [3]. Internal migration of the population for difficult economic reasons led to the establishment of peripheral areas of cities, which leads to favorable environments to the rapid spread of vectors. In addition, poverty rate is an indicator that entails bad nutrition, causing population to be more amenable to the pathogen [41]. Another study has shown that poverty can increase the risk of leishmaniasis due to poor hygienic conditions [41]. In our case, we were able to show another result; that is, poverty does not influence the distribution of the incidence of CL cases at the level of Sefrou Province but has an impact on the distribution of CL cases. A study in Quebec coincides with ours and it has been able to show that poverty does not explain the emergence of CL [42]. The same is true in Morocco, especially in Meknes Province, as the poverty rate of municipalities has no influence on the distribution of the epidemic [11].

\section{Conclusion}

Various factors can have an effect on the distribution of leishmaniasis. This work emphasizes the effect of urbanization and socioeconomic risks on the distribution of cases of cutaneous leishmaniasis at the level of Sefrou Province. The use of GIS is an added value to the creation of thematic maps which function as CL risk maps. These results must be taken into consideration in determining the risk zones for leishmaniasis and their risk factors in order to establish effective control strategies.

\section{Data Availability}

No data were used to support this study.

\section{Conflicts of Interest}

The authors declare that there are no conflicts of interest regarding the publication of this paper.

\section{References}

[1] J. Euzeby, Human Parasitic Diseases of Animal Origin, Epidemiological Characters, Vol. 324, Flammarion-MédecineScience, Paris, France, 1984.

[2] I. Parrot, A. Donatien, and E. Plantureux, "On the natural infection of sand flies by general leishmaniasis of humans and dogs in Algeria," Archives De L'institut Pasteur Algérie, vol. 19, pp. 209-217, 1941.

[3] WHO, Control of the Leishmaniasis. WHO Technical Report Series, p. 793, Repport of a WHO expert committee, Geneva, Switzerland, 2010.

[4] WHO, Continue to Act to Reduce the Global Impact of Neglected Tropical Diseases: Second WHO Report on Neglected Tropical Diseases, WHO, Geneva, Switzerland, 2010.

[5] H. W. Murray, "Kala-azar as an AIDS-related opportunistic infection," AIDS Patient Care and STDs, vol. 13, no. 8, pp. 459-465, 1999.

[6] Moroccan Ministry of Health, "Health in figures 2013," 2014, http://www.sante.gov.ma/Publications/Etudes_enquete/ Documents/Sant\%C3\%A9enchiffres2013edition2014-.pdf.

[7] A. Tabbabi, S. Sboui, and K. Bekhti, "A historical overview of the classification, evolution, and dispersion of Leishmania parasites and sandflies in Morocco," Journal of Infectious Diseases \& Therapy, vol. 5, no. 332, 2017.

[8] I. Laamrani, "Leishmaniasis control program in Morocco" National Seminar on Rodent Surveillance and Control, vol. 20, pp. 15-23, 2000.

[9] J. A. Rioux, P. Rispail, G. Lanotte, and J. Lepart, "Sandfly-bioclimate relationships in the ecology of epidemiological corollaries leishmaniasis, the example of Morocco," Bulletin of the Botanical Society of France, vol. 131, no. 2-4, pp. 549-557, 1984.

[10] M. Hakkour, A. Hmamouch, M. M. El Alem et al., "New epidemiological aspects of visceral and cutaneous leishmaniasis in Taza, Morocco," Parasites \& Vectors, vol. 9, no. 1, p. 612, 2016.

[11] H. El Miri, C. Faraj, O. Himmi et al., "Cutaneous leishmaniasis in ouazzane and Sidi Kacem provinces, Morocco (1997-2012)," Bulletin de la Société de pathologie exotique, vol. 109, no. 5, pp. 376-380, 2016.

[12] M. M. El Alem, F. Sebti, L. Med et al., "Geographical distribution and new situation of Leishmania species after the control of cutaneous leishmaniasis foci in Errachidia Province, Morocco in 2014," BioMed Research International, vol. 2016, Article ID 8642373, 7 pages, 2016.

[13] F. Z. Talbi, C. Faraj, F. EL-Akhal et al., "Diversity and dynamics of sand flies (diptera: psychodidae) of two cutaneous leishmaniasis foci in the fes-boulemane region of northern Morocco," International Journal of Zoology, vol. 2015, p. 6, 2015.

[14] F. Z. Talbi, A. El Ouali Lalami, A. Janati Idrissi, F. Sebti, and C. Faraj, "Leishmaniasis in central Morocco: seasonal fluctuations of phlebotomine sand fly in Aichoun locality, from Sefrou province," Pathology Research International, vol. 2015, p. 4, 2015.

[15] F. Z. Talbi, L. Aarab, C. Faraj, A. Janati Idrissi, and A. El Ouali Lalami, "Monitoring of vector-borne diseases: investigation of feeding preferences of the sand fly, Phlebotomus perniciosus (diptera: psychodidae) in a focus of cutaneous leishmaniasis in aichoun, north center of Morocco," International Journal of Pharmaceutical Sciences Review and Research, vol. 41, no. 2, pp. 48-52, 2016.

[16] F. Z. Talbi, A. Janati Idrissi, A. Sandoudi, and A. El Ouali Lalami, "Spatial distribution of incidence of leishmaniasis of 
different communes of Sefrou Province (2007--2010), central north of Morocco," in Proceedings of the 4th International Conference on Smart City Applications (SCA'19), Casablanca, Morocco, October 2019.

[17] R. González, L. D. Sousa, R. Devera, A. Jorquera, and E. Ledezma, "seasonal and nocturnal domiciliary human landing/biting behaviour of lutzomyia (lutzomyia) evansi and lutzomyia (psychodopygus) panamensis (diptera; psychodidae) in a periurban area of a city on the caribbean coast of eastern venezuela (Barcelona; Anzoátegui State)," Transactions of the Royal Society of Tropical Medicine and Hygiene, vol. 93, no. 4, pp. 361-364, 1999.

[18] H. El Omari, A. Chahlaoui, F. Talbi, K. Ouarrak, and A. El Ouali Lalami, "Impact of urbanization and socioeconomic factors on the distribution of cutaneous leishmaniasis in the center of Morocco," Interdisciplinary Perspectives on Infectious Diseases, vol. 2020, Article ID 2196418, 7 pages, 2020.

[19] K. E. Mott, P. Desjeux, A. Moncayo, P. Ranque, and P. Raadt, "Parasitoses et urbanisation," Bulletin de l'Organisation mondiale de la santé, vol. 69, no. 1, pp. 9-16, 1991.

[20] M. R. Shirzadi, M. Javanbakht, N. Jesri, and A. Saghafipour, "Spatial distribution of cutaneous leishmaniasis cases referred to health centers of three khorasan provinces in Iran using geographical information system," Iranian Journal of Public Health, vol. 48, no. 10, pp. 1885-1892, 2019.

[21] M. Salimi, N. Jesri, M. Javanbakht, L. Z. Farahani, M. R. Shirzadi, and A. Saghafipour, "Spatio-temporal distribution analysis of zoonotic cutaneous leishmaniasis in Qom Province, Iran," Journal of Parasitic Diseases, vol. 42, no. 4, pp. 570-576, 2018.

[22] High Commission for Planning (HCP), Regional Direction of Fès-Boulemane, Socio-Economic Monography of the FèsBoulemane Region, High Commission for Planning, Casablanca, Morocco, 2012.

[23] M. Rhajaoui, A. Nasereddin, H. Fellah et al., "New clinicoepidemiologic profile of cutaneous leishmaniasis, Morocco," Emerging Infectious Diseases, vol. 13, no. 9, pp. 1358-1360, 2007.

[24] F. Z. Talbi, F. El Khayyat, H. El Omari et al., "Cartography and epidemiological study of leishmaniasis disease in sefrou province (2007-2010)," Central North of Morocco, vol. 2020, Article ID 1867651, 8 pages, 2020.

[25] High Commission for Planning (HCP), General Census of Population and Housing, High Commission for Planning, Casablanca, Morocco, 2004.

[26] S. Boussaa and A. Boumezzough, "Entomologie faunistique - faunistic entomology," Identification Et Caractérisation Des Gîtes Larvaires De Phlébotomes (Diptera: Psychodidae) À Marrakech (Maroc), vol. 67, pp. 193-201, 2014.

[27] WHO, Leishmaniasis Control Report of the Meeting of the WHO Expert Committee on Leishmaniasis Control, World Health Organization, Geneva, Switzerland, 2011.

[28] H. El Omari, A. Chahlaoui, A. El Ouali Lalami, and M. khaffou, "The contribution of geographic information systems in the fight against parasitic diseases: the case of leishmaniasis," in Proceedings of the 3rd International Conference on Smart City Applications (SCA'18), B. A. Mohamed, B. A. Abdelhakim, and Y. Ali, Eds., October 2018.

[29] UN-Habitat, Urbanization Trends: Urban Sprawl Is Now a Global Problem, UN-Habitat, Nairobi, Kenya, 2010.

[30] WHO, The World Health Report, World Health Organization, Geneva, Switzerland, 2004.

[31] P. Desjeux, Leishmaniases, Public Health aspect and control, Vol. 253, Edition Ellipses, Paris, France, 1999.
[32] M. A. Ghatee, I. Sharifi, A. A. Haghdoost et al., "Spatial correlations of population and ecological factors with distribution of visceral leishmaniasis cases in southwestern Iran," Journal of Vector Borne Diseases, vol. 50, no. 3, pp. 179-187, 2013.

[33] P. Desjeux, The Increase in Risk Factors for Leishmaniasis Worldwide, WHO/Mediterranean Zoonoses Control Centre, Athens, Greece, 2002.

[34] R. Reithinger, M. Mohsen, and T. Leslie, "Risk factors for anthroponotic cutaneous leishmaniasis at the household level in Kabul, Afghanistan," PLoS Neglected Tropical Diseases, vol. 4, no. 3, p. e639, 2010.

[35] World Health Organization (WHO), "Urbanization: an increasing risk factor for leishmaniasis," The Weekly Epidemiological Record, vol. 77, no. 44, pp. 365-372, 2002.

[36] K. Kahime, S. Boussaa, A. Laamrani-El Idrissi, H. Nhammi, and A. Boumezzough, "Epidemiological study on acute cutaneous leishmaniasis in Morocco," Journal of Acute Disease, vol. 5, no. 1, pp. 41-45, 2016.

[37] K. Aoun, F. Jeddi, F. Amri, J. Ghrab, and A. Bouratbine, "Actualités épidémiologiques de la leishmaniose viscérale en Tunisie," Médecine et Maladies Infectieuses, vol. 39, no. 10, pp. 775-779, 2009.

[38] M. Rhajaoui, H. Fellah, F. Pratlong, J. P. Dedet, and M. Lyagoubi, "Leishmaniasis due to leishmania tropica Mon102 in a new Moroccan focus," Transactions of the Royal Society of Tropical Medicine and Hygiene, vol. 98, no. 5, pp. 299-301, 2004.

[39] K. Kahime, B. Bounoua, M. Messouli, S. Boussaa, and A. Boumezzough, "Evaluation of eco-adaptation strategies of health to climate change: case of zoonotic cutaneous leishmaniasis (ZCL) as vulnerability indicator in pre-Saharan region of Morocco," in Environmental Change and Human Security in the Middle East and Africa. GECS 2012 Conference Proceedings, M. Behnassi, Ed., pp. 117-131, Springer/Cambridge University Press, Cambridge, England, UK, 2017.

[40] J. Alvar, I. D. Velez, C. Bern et al., "Leishmaniasis worldwide and global estimates of its incidence," PLoS One, vol. 7, no. 5, Article ID e35671, 2012.

[41] A. Jorge, Y. Sergio, and B. Caryn, "Leishmaniasis and poverty," TRENDS in Parasitology, vol. 22, no. 12, pp. 552-557, 2006.

[42] Desplanques et Piovano, Leishmaniasis, Diseases without Borders, Quebec Science, 2016. 